\title{
An Asymptotic Nondegeneracy Result for a Biharmonic Equation with the Nearly Critical Growth
}

Futoshi Takahashi

\begin{abstract}
We consider the problem $\Delta^{2} u=c_{0} u^{p_{\varepsilon}}, u>0$ in $\Omega, u=\Delta u=0$ on $\partial \Omega$, where $\Omega$ is a smooth bounded domain in $\mathbb{R}^{N}(N \geq 5), c_{0}=(N-4)(N-$ 2) $N(N+2), p_{\varepsilon}=p-\varepsilon, p=(N+4) /(N-4)$ is the critical Sobolev exponent of the embedding $H^{2} \cap H_{0}^{1}(\Omega) \hookrightarrow L^{p+1}(\Omega)$.

We prove that along the solution sequence $\left\{u_{\varepsilon}\right\}$ minimizing the Sobolev quotient, each $u_{\varepsilon}$ is nondegenerate for $\varepsilon>0$ small, if the blow up point of $\left\{u_{\varepsilon}\right\}$ is a nondegenerate critical point of the Robin function. This is a $\Delta^{2}$ counterpart of the former work by Grossi [9] for the $-\Delta$ case problem, and it needs some new argument.
\end{abstract}

Mathematics Subject Classification (2000). 35B33, 35J35.

Keywords. Nondegeneracy, critical exponent, Robin function.

\section{Introduction}

In this paper, we consider the problem

$$
\left(P_{\varepsilon}\right) \begin{cases}\Delta^{2} u=c_{0} u^{p_{\varepsilon}} & \text { in } \Omega, \\ u>0 & \text { in } \Omega, \\ u=\Delta u=0 & \text { on } \partial \Omega\end{cases}
$$

where $\Omega \subset \mathbb{R}^{N}(N \geq 5)$ is a smooth bounded domain, $c_{0}=(N-4)(N-2) N(N+2)$, $\varepsilon>0$ is a small positive parameter, $p_{\varepsilon}=p-\varepsilon$ where $p=(N+4) /(N-4)$ is the critical Sobolev exponent from the view point of the Sobolev embedding $H^{2} \cap H_{0}^{1}(\Omega) \hookrightarrow L^{p+1}(\Omega)$. Here, $H^{2} \cap H_{0}^{1}(\Omega)$ is a Hilbert space whose norm is given by

$$
\|u\|_{H^{2} \cap H_{0}^{1}(\Omega)}=\left(\int_{\Omega}|\Delta u|^{2} d x\right)^{1 / 2} .
$$


Since $\left(P_{\varepsilon}\right)$ is a subcritical problem, the existence of solution for any $\varepsilon>0$ small is rather easy to obtain. Let $\left\{u_{\varepsilon}\right\}$ be a solution sequence which satisfies

$$
\frac{\left\|u_{\varepsilon}\right\|_{H^{2} \cap H_{0}^{1}(\Omega)}^{2}}{\left\|u_{\varepsilon}\right\|_{L^{p_{\varepsilon}+1}(\Omega)}^{2}} \rightarrow S_{N}, \quad \text { as } \quad \varepsilon \rightarrow 0,
$$

where $S_{N}$ is the best constant of the embedding $H^{2} \cap H_{0}^{1}(\Omega) \hookrightarrow L^{p+1}(\Omega)$ :

$$
S_{N}=\inf _{u \in H^{2} \cap H_{0}^{1}(\Omega)} \frac{\int_{\Omega}|\Delta u|^{2} d x}{\left(\int_{\Omega}|u|^{p+1} d x\right)^{2 /(p+1)}} .
$$

When $\Omega$ is strictly convex, Chou and Geng [4] proved the precise asymptotic behavior of positive solutions of $\left(P_{\varepsilon}\right)$ satisfying (1.1). Among other things, they proved that $\left\{u_{\varepsilon}\right\}$ blows up at exactly one point which is in the interior of $\Omega$, and the blow up point has to be a critical point of the Robin function associated to the Green function of $\Delta^{2}$ under the Navier boundary condition. Later, by using another method, Ben Ayed and El Mehdi [2], El Mehdi [6], Geng [8] succeeded to prove that the same result holds true without the convexity assumption of the domain.

In this article, we concern some qualitative property of the blowing-up solution $u_{\varepsilon}$. Recall that a solution $u$ of $\left(P_{\varepsilon}\right)$ is said to be nondegenerate, if the linearized problem around $u$ :

$$
\left(L_{\varepsilon}\right) \begin{cases}\Delta^{2} v=c_{0} p_{\varepsilon} u^{p_{\varepsilon}-1} v & \text { in } \quad \Omega \\ v=\Delta v=0 & \text { on } \partial \Omega\end{cases}
$$

admits only the trivial solution $v \equiv 0$.

Theorem 1.1. Let $\Omega \subset \mathbb{R}^{N}(N \geq 5)$ be a bounded smooth domain. Let $\left\{u_{\varepsilon}\right\}$ be a solution sequence to $\left(P_{\varepsilon}\right)$ satisfying (1.1) and let $x_{0}$ be the blow up point of $\left\{u_{\varepsilon}\right\}$. If $x_{0}$ is a nondegenerate critical point of the Robin function, then $u_{\varepsilon}$ is nondegenerate for $\varepsilon>0$ sufficiently small.

Grossi [9] considered the solutions $\left\{u_{\varepsilon}\right\}$ to

$$
\begin{cases}-\Delta u=N(N-2) u^{(N+2) /(N-2)-\varepsilon} & \text { in } \Omega, \\ u>0 & \text { in } \Omega, \\ u=0 & \text { on } \partial \Omega\end{cases}
$$

satisfying

$$
\frac{\left\|\nabla u_{\varepsilon}\right\|_{L^{2}(\Omega)}^{2}}{\left\|u_{\varepsilon}\right\|_{L^{2 N /(N-2)-\varepsilon}(\Omega)}^{2}} \rightarrow S_{N}, \quad \text { as } \quad \varepsilon \rightarrow 0 .
$$

Here $\Omega \subset \mathbb{R}^{N}(N \geq 3)$ is a smooth bounded domain and $S_{N}$ is the best constant of the embedding $H_{0}^{1}(\Omega) \hookrightarrow L^{2 N /(N-2)}(\Omega)$. Under the assumption that the blow up point of $\left\{u_{\varepsilon}\right\}$ is a nondegenerate critical point of the Robin function associated to the Green function of $-\Delta$ under the Dirichlet boundary condition, Grossi [9] proved the same nondegeneracy result for $\varepsilon>0$ small. 
Theorem 1.1 can be regarded as an extension of Grossi's theorem to the biharmonic case. However, some new arguments are needed to prove the result, which are not in [9]. Our argument also works well for the $-\Delta$ case problem, so it gives another proof of the result in [9].

\section{Preliminaries}

In this section, we recall some useful facts. Let $G=G(x, z)$ denote the Green function of $\Delta^{2}$ under the Navier boundary condition:

$$
\begin{cases}\Delta^{2} G(\cdot, z)=\delta_{z} & \text { in } \Omega, \\ G(\cdot, z)=\Delta G(\cdot, z)=0 & \text { on } \partial \Omega .\end{cases}
$$

We decompose $G$ as $G(x, z)=\Gamma(x, z)-H(x, z)$, where $\Gamma(x, z)$ is the fundamental solution of $\Delta^{2}$ :

$$
\Gamma(x, z)= \begin{cases}\frac{1}{(N-4)(N-2) \sigma_{N}}|x-z|^{4-N}, & N \geq 5, \\ \frac{1}{\sigma_{4}} \log |x-z|^{-1}, & N=4\end{cases}
$$

where $\sigma_{N}$ is the volume of the $(N-1)$ dimensional unit sphere in $\mathbb{R}^{N}$ and $H(x, z)$ is the regular part of the Green function. Finally, let $R(x)=H(x, x)$ denote the Robin function of the Green function of $\Delta^{2}$ with the Navier boundary condition. By the maximum principle, we have $R>0$ on $\Omega$ and $R(x) \rightarrow+\infty$ as $x$ tends to the boundary of $\Omega$. In the following, we set $\bar{G}=-\Delta G$. Then $\bar{G}$ is the Green function of $-\Delta$ under the Dirichlet boundary condition, and satisfy

$$
\begin{cases}-\Delta G=\bar{G} & \text { in } \Omega, \\ -\Delta \bar{G}=\delta_{z} & \text { in } \Omega, \\ G>0, \bar{G}>0 & \text { in } \quad \Omega, \\ G=\bar{G}=0 & \text { on } \quad \partial \Omega .\end{cases}
$$

Lemma 2.1 (Pohozaev identity for the Green function).

$$
\int_{\partial \Omega}\left((x-z) \cdot \nu_{x}\right)\left(\frac{\partial G}{\partial \nu_{x}}\right)\left(\frac{\partial \bar{G}}{\partial \nu_{x}}\right)(x, z) d s_{x}=(N-4) R(z)
$$

and

$$
\int_{\partial \Omega} \frac{\partial G}{\partial \nu_{x}}(x, z) \frac{\partial \bar{G}}{\partial \nu_{x}}(x, z) \nu_{i}(x) d s_{x}=\frac{\partial R}{\partial z_{i}}(z) \quad(i=1, \ldots, N)
$$

hold true for any $z \in \Omega$. Here $\nu_{x}$ is the outer unit normal at $x \in \partial \Omega$.

Proof. See [4, Lemma 3.1 and Lemma 3.3]. Note that our sign convention is different from that of [4]. Also note that there is a mistake in the claimed formula in [4]. 
Lemma 2.2.

$$
\begin{aligned}
\frac{\partial^{2} R}{\partial z_{i} \partial z_{j}}(z)= & \int_{\partial \Omega}\left(\frac{\partial \bar{G}}{\partial x_{i}}\right)(x, z) \frac{\partial}{\partial z_{j}}\left(\frac{\partial G}{\partial \nu_{x}}\right)(x, z) d s_{x} \\
& +\int_{\partial \Omega}\left(\frac{\partial G}{\partial x_{i}}\right)(x, z) \frac{\partial}{\partial z_{j}}\left(\frac{\partial \bar{G}}{\partial \nu_{x}}\right)(x, z) d s_{x}
\end{aligned}
$$

holds true for any $z \in \Omega$ and for $1 \leq i, j \leq N$.

Proof. Differentiating (2.3) with respect to $z_{j}$, noting that $\left(\frac{\partial G}{\partial \nu_{x}}(x, z)\right) \nu_{i}(x)=$ $\frac{\partial G}{\partial x_{i}}(x, z),\left(\frac{\partial \bar{G}}{\partial \nu_{x}}(x, z)\right) \nu_{i}(x)=\frac{\partial \bar{G}}{\partial x_{i}}(x, z)$ on $\partial \Omega$, we obtain the result. equation.

Next lemma is a classical elliptic regularity for a solution to the biharmonic

Lemma 2.3. Let $u \in H_{\text {loc }}^{2}(\Omega)$ be a weak solution of

$$
\Delta^{2} u=a(x) u \text { in } \Omega
$$

where $a \in L^{\alpha}(\Omega)$ with $\alpha>N / 4$. Then for any $q \in(0,+\infty)$, there exist $C=$ $C(q)>0$ and $R>0$ such that for any $0<r<R$ and $y \in \mathbb{R}^{N}$,

$$
\sup _{B(y, r) \cap \Omega}|u| \leq C\left[\frac{1}{r^{N}} \int_{B(y, 2 r) \cap \Omega}|u|^{q+1} d x\right]^{1 /(q+1)}
$$

holds true.

Proof. See [3].

Lemma 2.4. Let $u_{\varepsilon}$ be a solution to $\left(P_{\varepsilon}\right)$ and $v_{\varepsilon}$ be a solution to $\left(L_{\varepsilon}\right)$ with $u=u_{\varepsilon}$. Denote $\bar{u}_{\varepsilon}=-\Delta u_{\varepsilon}$ and $\bar{v}_{\varepsilon}=-\Delta v_{\varepsilon}$. Then the following identities hold true:

$$
\int_{\partial \Omega}\left((x-z) \cdot \nu_{x}\right)\left\{\left(\frac{\partial u_{\varepsilon}}{\partial \nu_{x}}\right)\left(\frac{\partial \bar{v}_{\varepsilon}}{\partial \nu_{x}}\right)+\left(\frac{\partial \bar{u}_{\varepsilon}}{\partial \nu_{x}}\right)\left(\frac{\partial v_{\varepsilon}}{\partial \nu_{x}}\right)\right\} d s_{x}=0
$$

for any $z \in \mathbb{R}^{N}$ and

$$
\int_{\partial \Omega}\left\{\left(\frac{\partial \bar{u}_{\varepsilon}}{\partial x_{i}}\right)\left(\frac{\partial v_{\varepsilon}}{\partial \nu_{x}}\right)+\left(\frac{\partial u_{\varepsilon}}{\partial x_{i}}\right)\left(\frac{\partial \bar{v}_{\varepsilon}}{\partial \nu_{x}}\right)\right\} d s_{x}=0, \quad i=1,2, \ldots, N .
$$

Proof. For smooth $f, g$, we have the formula

$$
\begin{aligned}
\int_{\Omega} & \left(\left(\Delta^{2} f\right) g-\left(\Delta^{2} g\right) f\right) d x \\
\quad= & \int_{\partial \Omega}\left(\frac{\partial \Delta f}{\partial \nu_{x}}\right) g-\left(\frac{\partial \Delta g}{\partial \nu_{x}}\right) f d s_{x}+\int_{\partial \Omega}\left(\frac{\partial f}{\partial \nu_{x}}\right) \Delta g-\left(\frac{\partial g}{\partial \nu_{x}}\right) \Delta f d s_{x} .
\end{aligned}
$$

Set $w_{\varepsilon}(x)=(x-z) \cdot \nabla u_{\varepsilon}(x)+\alpha_{\varepsilon} u_{\varepsilon}(x)$ where $\alpha_{\varepsilon}=\frac{4}{p_{\varepsilon}-1}$. Direct computation yields that

$$
\Delta^{2} w_{\varepsilon}=\left(\alpha_{\varepsilon}+4\right) c_{0} u_{\varepsilon}^{p_{\varepsilon}}+c_{0} p_{\varepsilon} u_{\varepsilon}^{p_{\varepsilon}-1}(x-z) \cdot \nabla u_{\varepsilon} .
$$

Since $v_{\varepsilon}$ satisfies $\Delta^{2} v_{\varepsilon}=c_{0} p_{\varepsilon} u_{\varepsilon}^{p_{\varepsilon}-1} v_{\varepsilon}$, we have

$$
\left(\Delta^{2} w_{\varepsilon}\right) v_{\varepsilon}-\left(\Delta^{2} v_{\varepsilon}\right) w_{\varepsilon}=\left(\alpha_{\varepsilon}+4-p_{\varepsilon} \alpha_{\varepsilon}\right) c_{0} u_{\varepsilon}^{p_{\varepsilon}} v_{\varepsilon}=0
$$


Integrating this identity on $\Omega$ with the formula (2.7), and noting that

$$
w_{\varepsilon}(x)=(x-z) \cdot \nu_{x}\left(\frac{\partial u_{\varepsilon}}{\partial \nu_{x}}\right), \quad \Delta w_{\varepsilon}(x)=(x-z) \cdot \nu_{x}\left(\frac{\partial \Delta u_{\varepsilon}}{\partial \nu_{x}}\right)
$$

for $x \in \partial \Omega$, we have (2.5).

On the other hand, differentiating the equation in $\left(P_{\varepsilon}\right)$ with respect to $x_{i}$ we have

$$
\Delta^{2}\left(\frac{\partial u_{\varepsilon}}{\partial x_{i}}\right)=c_{0} p_{\varepsilon} u_{\varepsilon}^{p_{\varepsilon}-1}\left(\frac{\partial u_{\varepsilon}}{\partial x_{i}}\right) \quad \text { in } \Omega .
$$

Multiplying this by $v_{\varepsilon}$, and the equation of $v_{\varepsilon}$ by $\left(\frac{\partial u_{\varepsilon}}{\partial x_{i}}\right)$ and subtracting, we obtain

$$
\left(\Delta^{2} v_{\varepsilon}\right)\left(\frac{\partial u_{\varepsilon}}{\partial x_{i}}\right)-\left(\Delta^{2}\left(\frac{\partial u_{\varepsilon}}{\partial x_{i}}\right)\right) v_{\varepsilon}=0 .
$$

Finally, integration by parts formula (2.7) yields (2.6).

Next is the main asymptotic result by Chou and Geng [4].

Theorem 2.5 ([4]). Assume $\Omega \subset \mathbb{R}^{N}, N \geq 5$ is strictly convex. Let $u_{\varepsilon}$ be a solution to $\left(P_{\varepsilon}\right)$ satisfying $(1.1)$. Let $x_{\varepsilon} \in \Omega$ be a point such that $u_{\varepsilon}\left(x_{\varepsilon}\right)=\left\|u_{\varepsilon}\right\|_{L^{\infty}(\Omega)}$. Then after passing to a subsequence, we have

(1) $\lim _{\varepsilon \rightarrow 0} x_{\varepsilon}=x_{0}$ for some interior point $x_{0} \in \Omega$. Furthermore, $x_{0}$ is a critical point of the Robin function $R$, i.e., $\nabla R\left(x_{0}\right)=0$.

(2) There holds

$$
\left\|u_{\varepsilon}\right\|_{L^{\infty}(\Omega)} u_{\varepsilon} \rightarrow 2(N-4)(N-2) \sigma_{N} G\left(\cdot, x_{0}\right) \quad \text { in } \quad C_{l o c}^{3}\left(\bar{\Omega} \backslash\left\{x_{0}\right\}\right)
$$

(3)

as $\varepsilon \rightarrow 0$.

$$
\lim _{\varepsilon \rightarrow 0}\left\|u_{\varepsilon}\right\|_{L^{\infty}(\Omega)}^{\varepsilon}=1
$$

(4) There exists a constant $C>0$ independent of $\varepsilon$ such that

$$
u_{\varepsilon}(x) \leq C \frac{\left\|u_{\varepsilon}\right\|_{L^{\infty}(\Omega)}}{\left(1+\left\|u_{\varepsilon}\right\|_{L^{\infty}(\Omega)}^{\frac{4}{N-4}}\left|x-x_{\varepsilon}\right|^{2}\right)^{\frac{N-4}{2}}}
$$

holds for any $x \in \Omega$.

(5)

$$
\lim _{\varepsilon \rightarrow 0} \varepsilon\left\|u_{\varepsilon}\right\|_{L^{\infty}(\Omega)}^{2}=\frac{16 \sigma_{N}^{2} \Gamma(N) c_{0}}{\pi^{N / 2} N(N-4)(N+2)^{2} \Gamma(N / 2)} R\left(x_{0}\right) .
$$

Remark. In [4], the assumption of the strict convexity of the domain is used in twofold: One is to exclude the possible boundary blow-ups and to obtain uniform $L^{\infty}$-estimate for solutions near the boundary by the method of moving planes, and the other is to obtain the pointwise estimate (2.10) by using the Kelvin transformation.

Now, thanks to the works of Ben Ayed and El Mehdi [2], El Mehdi [6] and Geng [8], we know that the blow up point $x_{0}$ for $u_{\varepsilon}$ satisfying (1.1) must be 
an interior point of $\Omega$ and is a critical point of the Robin function, without the convexity assumption of the domain. Therefore $u_{\varepsilon}$ satisfying (1.1) is uniformly bounded near the boundary.

Also we can prove the pointwise estimate (2.10) via the theory of isolated simple blow up points performed by Geng [7]. For definitions of isolated, and isolated simple blow up points, we refer to [7]. We also refer to the original work by YanYan Li [10] for $-\Delta$ case problem. Though strict convexity of the domain is assumed in [7], it is simply because Geng had to treat solution sequences without energy bounds, which may blow up on the boundary. Thus if we a priori know that the blow up points of $\left\{u_{\varepsilon}\right\}$ are interior points of the domain, convexity assumption is not needed in applying the results in [7]. It is proved in [7] that the interior blow up points have to be isolated simple ones. Therefore we derive (2.10) from the estimates for isolated simple blow up points in [7].

In conclusion, we confirm that any convexity assumption is not needed for Theorem 2.5, and consequently, for Theorem 1.1 to hold.

In what follows, we use a symbol $\|\cdot\|$ to denote the $L^{\infty}$ norm of functions. Now, let us consider the scaled function

$$
\tilde{u}_{\varepsilon}(y):=\frac{1}{\left\|u_{\varepsilon}\right\|} u_{\varepsilon}\left(\frac{y}{\left\|u_{\varepsilon}\right\|^{\frac{p_{\varepsilon}-1}{4}}}+x_{\varepsilon}\right), \quad y \in \Omega_{\varepsilon}:=\left\|u_{\varepsilon}\right\|^{\frac{p_{\varepsilon}-1}{4}}(\Omega-\varepsilon) .
$$

We see $0<\tilde{u}_{\varepsilon} \leq 1, \tilde{u}_{\varepsilon}(0)=1$, and $\tilde{u}_{\varepsilon}$ satisfies

$$
\left\{\begin{array}{cl}
\Delta^{2} \tilde{u}_{\varepsilon}=c_{0} \tilde{u}_{\varepsilon}^{p_{\varepsilon}} & \text { in } \quad \Omega_{\varepsilon} \\
\tilde{u}_{\varepsilon}=\Delta \tilde{u}_{\varepsilon}=0 & \text { on } \quad \partial \Omega_{\varepsilon} .
\end{array}\right.
$$

Since $\left\|u_{\varepsilon}\right\| \rightarrow \infty$ as $\varepsilon \rightarrow 0$ and $x_{\varepsilon}$ does not approach to $\partial \Omega$, we see $\Omega_{\varepsilon} \rightarrow \mathbb{R}^{N}$. By standard elliptic estimates, we have a subsequence denoted also by $\tilde{u}_{\varepsilon}$ that

$$
\tilde{u}_{\varepsilon} \rightarrow U \text { compact uniformly in } \mathbb{R}^{N}
$$

as $\varepsilon \rightarrow 0$ for some function $U$. Passing to the limit, we obtain that $U$ is a solution of

$$
\left\{\begin{array}{l}
\Delta^{2} U=c_{0} U^{p} \quad \text { in } \quad \mathbb{R}^{N} \\
0<U \leq 1, \quad U(0)=1 \\
\lim _{|y| \rightarrow \infty} U(y)=0
\end{array}\right.
$$

Thus according to the uniqueness theorem by Chang Shou Lin [11], we obtain

$$
U(y)=\left(\frac{1}{1+|y|^{2}}\right)^{\frac{N-4}{2}} .
$$

Also by (2.9), (2.10) can be written as

$$
\tilde{u}_{\varepsilon}(y) \leq C U(y) \text { for } y \in \Omega_{\varepsilon} .
$$

Now, we recall the classification theorem by Bartsch, Weth and Willem [1]. 
Lemma 2.6. Let $v_{0}$ be a solution to

$$
\left\{\begin{array}{l}
\Delta^{2} v_{0}=c_{0} p U^{p-1} v_{0} \quad \text { in } \quad \mathbb{R}^{N}, \\
\int_{\mathbb{R}^{N}}\left|\Delta v_{0}\right|^{2} d y \leq C .
\end{array}\right.
$$

Then there exist $a_{j}(j=1,2, \ldots, N), b \in \mathbb{R}$ such that $v_{0}$ can be written as

$$
v_{0}=\sum_{j=1}^{N} a_{j} \frac{y_{j}}{\left(1+|y|^{2}\right)^{(N-2) / 2}}+b \frac{1-|y|^{2}}{\left(1+|y|^{2}\right)^{(N-2) / 2}} .
$$

Next lemma is a unique solvability result for linear first order PDE's with the initial condition, and is well known.

Lemma 2.7. Let $a=\left(a_{1}, a_{2}, \ldots, a_{N}\right) \neq 0$ is a constant vector and $f, g \in C^{1}\left(\mathbb{R}^{N}\right)$. Let $\Gamma_{a}=\left\{x \in \mathbb{R}^{N} \mid a \cdot x=0\right\}$ be the $(N-1)$-plane perpendicular to a through the origin. Then there exists a unique solution of the following initial value problem of the linear first order PDE

$$
\begin{aligned}
a \cdot \nabla u & =f, \\
\left.u\right|_{\Gamma_{a}} & =g .
\end{aligned}
$$

More precisely, this solution is obtained as

$$
u(x)=\int_{0}^{\phi(x)} f(\tau a+\alpha(\psi(x))) d \tau+g(\alpha(\psi(x))), \quad x \in \mathbb{R}^{N}
$$

where

$$
\begin{aligned}
\phi(x) & =\frac{a \cdot x}{|a|^{2}}, \quad \psi(x)=\left(\psi_{1}(x), \ldots, \psi_{N-1}(x)\right), \\
\psi_{j}(x) & =\frac{|a|^{2} x_{j}-(a \cdot x) a_{j}}{|a|^{2}}, \quad(j=1, \ldots, N-1) \\
\alpha(s) & =\left(s,-\frac{1}{a_{N}} \sum_{j=1}^{N-1} a_{j} s_{j}\right) \in \mathbb{R}^{N}, \quad s=\left(s_{1}, \ldots, s_{N-1}\right) \in \mathbb{R}^{N-1},
\end{aligned}
$$

if we assume (w.l.o.g) $a_{N} \neq 0$. Furthermore, if $f(x)=O\left(|x|^{\beta}\right), g(x)=O\left(|x|^{\beta}\right)$ as $|x| \rightarrow \infty$, then $u(x)=O\left(|x|^{\beta+1}\right)$ as $|x| \rightarrow \infty$.

Proof. See, for example, [12].

\section{Proof of Theorem 1.1}

In this section, we will prove Theorem 1.1. As noticed earlier, we mainly follow the argument by Grossi [9], but some new argument is needed.

We argue by contradiction. We assume there exists a non-trivial solution $v_{\varepsilon}$ to $\left(L_{\varepsilon}\right)$ with $u=u_{\varepsilon}$ satisfying $\left\|v_{\varepsilon}\right\|=\left\|u_{\varepsilon}\right\|$ for any $\varepsilon>0$. 
Let us consider the scaled function

$$
\tilde{v}_{\varepsilon}(y):=\frac{1}{\left\|u_{\varepsilon}\right\|} v_{\varepsilon}\left(\frac{y}{\left\|u_{\varepsilon}\right\|^{\frac{p_{\varepsilon}-1}{4}}}+x_{\varepsilon}\right), \quad y \in \Omega_{\varepsilon}=\left\|u_{\varepsilon}\right\|^{\frac{p_{\varepsilon}-1}{4}}\left(\Omega-x_{\varepsilon}\right) .
$$

We see $0<\tilde{v}_{\varepsilon} \leq 1$ and $\tilde{v}_{\varepsilon}$ satisfies

$$
\begin{cases}\Delta^{2} \tilde{v}_{\varepsilon}=c_{0} p_{\varepsilon} \tilde{u}_{\varepsilon}^{p_{\varepsilon}-1} \tilde{v}_{\varepsilon} & \text { in } \quad \Omega_{\varepsilon} \\ \tilde{v}_{\varepsilon}=\Delta \tilde{v}_{\varepsilon}=0 & \text { on } \partial \Omega_{\varepsilon} \\ \left\|\tilde{v}_{\varepsilon}\right\|_{L^{\infty}\left(\Omega_{\varepsilon}\right)}=1 & \end{cases}
$$

By $\left\|\tilde{v}_{\varepsilon}\right\|_{L^{\infty}\left(\Omega_{\varepsilon}\right)}=1$ and the elliptic estimate, we see there exists $v_{0}$ such that

$$
\tilde{v}_{\varepsilon} \rightarrow v_{0} \text { compact uniformly in } \mathbb{R}^{N}
$$

and $v_{0}$ satisfies

$$
\Delta^{2} v_{0}=c_{0} p U^{p-1} v_{0} \quad \text { in } \quad \mathbb{R}^{N} .
$$

Now, we claim that

$$
\int_{\Omega_{\varepsilon}}\left|\Delta \tilde{v}_{\varepsilon}\right|^{2} d y \leq C
$$

for some $C>0$ independent of $\varepsilon>0$ small.

Set

$$
c_{\varepsilon}(y):=c_{0} p_{\varepsilon} \tilde{u}_{\varepsilon}^{p_{\varepsilon}-1}(y), \quad y \in \Omega_{\varepsilon} .
$$

By the Sobolev inequality and (3.2), we have

$$
S_{N}\left(\int_{\Omega_{\varepsilon}}\left|\tilde{v}_{\varepsilon}\right|^{p+1} d y\right)^{2 /(p+1)} \leq \int_{\Omega_{\varepsilon}}\left|\Delta \tilde{v}_{\varepsilon}\right|^{2} d y=\int_{\Omega_{\varepsilon}} c_{\varepsilon}(y) \tilde{v}_{\varepsilon}^{2} d y \leq \int_{\Omega_{\varepsilon}}\left|c_{\varepsilon}(y) \| \tilde{v}_{\varepsilon}^{2-\delta}\right| d y
$$

for some $0<\delta<\min (2,8 /(N-4)-2 \varepsilon)$, because $\left\|\tilde{v}_{\varepsilon}\right\| \leq 1$. Now, by the Hölder inequality and (2.14), we have

$$
\begin{aligned}
\int_{\Omega_{\varepsilon}}\left|c_{\varepsilon}(y)\right|\left|\tilde{v}_{\varepsilon}^{2-\delta}\right| d y \leq & \left(\int_{\Omega_{\varepsilon}}\left|\tilde{v}_{\varepsilon}\right|^{p+1} d y\right)^{\frac{(2-\delta)}{(p+1)}}\left(\int_{\Omega_{\varepsilon}}\left|c_{\varepsilon}(y)\right|^{(p+1) /(p-1+\delta)}\right)^{\frac{(p-1+\delta)}{(p+1)}} \\
\leq & C\left(\int_{\Omega_{\varepsilon}}\left|\tilde{v}_{\varepsilon}\right|^{p+1} d y\right)^{\frac{(2-\delta)}{(p+1)}} \\
& \times\left(\int_{\Omega_{\varepsilon}}(U(y))^{\left(p_{\varepsilon}-1\right)(p+1) /(p-1+\delta)}\right)^{\frac{(p-1+\delta)}{(p+1)}}
\end{aligned}
$$

thus we obtain

$$
\left(\int_{\Omega_{\varepsilon}}\left|\tilde{v}_{\varepsilon}\right|^{p+1} d y\right)^{\delta /(p+1)} \leq\left(\int_{\mathbb{R}^{N}}(U(y))^{\left(p_{\varepsilon}-1\right)(p+1) /(p-1+\delta)}(y)\right)^{(p-1+\delta) /(p+1)} .
$$


Note that $\left(p_{\varepsilon}-1\right)\left(\frac{N-4}{2}\right) \frac{p+1}{p-1+\delta}>N / 2$ if $\delta<8 /(N-4)-2 \varepsilon$, so the last integral is bounded by a constant. Finally, again (3.2) and the Hölder inequality, we have

$$
\begin{aligned}
\int_{\Omega_{\varepsilon}}\left|\Delta \tilde{v}_{\varepsilon}\right|^{2} d y & =\int_{\Omega_{\varepsilon}} c_{\varepsilon}(y) \tilde{v}_{\varepsilon}^{2} d y \\
& \leq\left(\int_{\Omega_{\varepsilon}}\left|\tilde{v}_{\varepsilon}\right|^{p+1} d y\right)^{2 /(p+1)}\left(\int_{\Omega_{\varepsilon}}\left|c_{\varepsilon}(y)\right|^{(p+1) /(p-1)} d y\right)^{(p-1) /(p+1)} \\
& \leq C\left(\int_{\mathbb{R}^{N}}(U(y))^{\left(p_{\varepsilon}-1\right)(p+1) /(p-1)} d y\right)^{(p-1) /(p+1)} \leq C
\end{aligned}
$$

if $\varepsilon>0$ is small so that $\left(\frac{N-4}{2}\right)\left(p_{\varepsilon}-1\right)(p+1) /(p-1)>N / 2$, i.e. $0<\varepsilon<4 /(N-4)$. This proves (3.4).

By (3.4) and Fatou's lemma, we also have

$$
\int_{\mathbb{R}^{N}}\left|\Delta v_{0}\right|^{2} d y \leq C
$$

Thus by Lemma 2.6, we have

$$
v_{0}=\sum_{j=1}^{N} a_{j} \frac{y_{j}}{\left(1+|y|^{2}\right)^{(N-2) / 2}}+b \frac{1-|y|^{2}}{\left(1+|y|^{2}\right)^{(N-2) / 2}} .
$$

In the following, we divide the proof into several steps.

Step 1. $b=0$.

Step 2. $a_{j}=0, j=1, \ldots, N$.

Step 3. $v_{0}=0$ leads to a contradiction.

We need the following pointwise estimate for the scaled function $\tilde{v}_{\varepsilon}$.

Lemma 3.1. Let $\tilde{v}_{\varepsilon}$ be a solution of (3.2). Then we have the estimate

$$
\left|\tilde{v}_{\varepsilon}(y)\right| \leq C U(y), \quad \forall y \in \Omega_{\varepsilon}
$$

for some $C>0$.

Proof. Consider the Kelvin transformation of $\tilde{v}_{\varepsilon}$ :

$$
z_{\varepsilon}(z)=|z|^{4-N} \tilde{v}_{\varepsilon}\left(\frac{z}{|z|^{2}}\right), \quad z \in \Omega_{\varepsilon}^{*}:=\left\{\frac{y}{|y|^{2}}: y \in \Omega_{\varepsilon}\right\} .
$$

Since $\left|\tilde{v}_{\varepsilon}(y)\right| \leq 1$ for any $y \in \Omega_{\varepsilon}$, it will be enough to show that $\left|z_{\varepsilon}\right|$ is bounded in $B(0, R) \cap \Omega_{\varepsilon}^{*}$ for some $R>0$ to prove (3.7). Direct calculation shows that

$$
\begin{aligned}
\Delta_{z}^{2} z_{\varepsilon}(z) & =|z|^{-4-N} \Delta_{y}^{2} \tilde{v}_{\varepsilon}(y), \quad z \in \Omega_{\varepsilon}^{*}, \\
\Delta_{z} z_{\varepsilon}(z) & =4|z|^{-2}\left(z, \nu_{z}\right) \frac{\partial z_{\varepsilon}}{\partial \nu_{z}}, \quad z \in \partial \Omega_{\varepsilon}^{*}, \\
\int_{\Omega_{\varepsilon}^{*}}\left|z_{\varepsilon}\right|^{p+1} d z & =\int_{\Omega_{\varepsilon}}\left|\tilde{v}_{\varepsilon}\right|^{p+1} d y .
\end{aligned}
$$


Thus, $z_{\varepsilon}$ satisfies the equation

$$
\left\{\begin{array}{lll}
\Delta^{2} z_{\varepsilon}=|z|^{-8} c_{\varepsilon}\left(\frac{z}{|z|^{2}}\right) z_{\varepsilon} & \text { in } & \Omega_{\varepsilon}^{*} \\
z_{\varepsilon}=0 & \text { on } & \partial \Omega_{\varepsilon}^{*} \\
\Delta z_{\varepsilon}=4|z|^{-2}\left(z, \nu_{z}\right) \frac{\partial z_{\varepsilon}}{\partial \nu_{z}}, & \text { on } & \partial \Omega_{\varepsilon}^{*}
\end{array}\right.
$$

where $c_{\varepsilon}$ is in (3.5). Now, we claim that

$$
a_{\varepsilon}(z):=|z|^{-8} c_{\varepsilon}\left(\frac{z}{|z|^{2}}\right) \in L^{\infty}\left(\Omega_{\varepsilon}^{*}\right) .
$$

Indeed, since $\Omega_{\varepsilon} \subset B\left(0, \gamma\left\|u_{\varepsilon}\right\|^{\left(p_{\varepsilon}-1\right) / 4}\right)$ for some $\gamma>0$, we see that the domain $\Omega_{\varepsilon}^{*}$ satisfies $\Omega_{\varepsilon}^{*} \subset \mathbb{R}^{N} \backslash B\left(0, \frac{1}{\gamma\left\|u_{\varepsilon}\right\|^{\left(p_{\varepsilon}-1\right) / 4}}\right)$. By (2.14), we have

$$
\left|c_{\varepsilon}(y)\right| \leq C U^{p_{\varepsilon}-1}(y) \text { for } y \in \Omega_{\varepsilon} .
$$

Therefore, we have

$$
\begin{aligned}
|z|^{-8} c_{\varepsilon}\left(\frac{z}{|z|^{2}}\right) & \leq C|z|^{-8}\left(\frac{|z|^{2}}{1+|z|^{2}}\right)^{\left(\frac{N-4}{2}\right)\left(p_{\varepsilon}-1\right)} \\
& =C|z|^{-8+(N-4)\left(p_{\varepsilon}-1\right)} \frac{1}{\left(1+|z|^{2}\right)^{4-\varepsilon\left(\frac{N-4}{2}\right)}} \\
& \leq C|z|^{-8+(N-4)\left(p_{\varepsilon}-1\right)}=C|z|^{-\varepsilon(N-4)} .
\end{aligned}
$$

Since $|z| \geq 1 /\left(\gamma\left\|u_{\varepsilon}\right\|^{\left(p_{\varepsilon}-1\right) / 4}\right)$ for $z \in \Omega_{\varepsilon}^{*}$, we have

$$
|z|^{-\varepsilon(N-4)} \leq \gamma^{\varepsilon(N-4)}\left\|u_{\varepsilon}\right\|^{\varepsilon(N-4)\left(p_{\varepsilon}-1\right) / 4} \rightarrow 1
$$

as $\varepsilon \rightarrow 0$. Here we have used (2.9). From these, we confirm the claim (3.10).

Now, for any $R>0$, we have

$\int_{\Omega_{\varepsilon}^{*} \cap B(0,2 R)}\left|z_{\varepsilon}\right|^{p+1} d z \leq \int_{\Omega_{\varepsilon}^{*}}\left|z_{\varepsilon}\right|^{p+1} d z=\int_{\Omega_{\varepsilon}}\left|\tilde{v}_{\varepsilon}\right|^{p+1} d y \leq\left(\frac{1}{S_{N}} \int_{\Omega_{\varepsilon}}\left|\Delta \tilde{v}_{\varepsilon}\right|^{2} d y\right)^{\frac{P+1}{2}} \leq C$ by (3.4). Let us take $q=p, y=0, \Omega=\Omega_{\varepsilon}^{*}$ in Lemma 2.3. Thus for $R>0$ in Lemma 2.3, we obtain

$$
\sup _{B(0, R) \cap \Omega_{\varepsilon}^{*}}\left|z_{\varepsilon}\right| \leq C\left[\frac{1}{R^{N}} \int_{B(0,2 R) \cap \Omega}\left|z_{\varepsilon}\right|^{p+1} d z\right]^{1 /(p+1)} \leq C .
$$
result.

By Lemma 3.1 and Theorem 2.5 (2.10), we have the following convergence

Lemma 3.2. Let $\omega \subset \Omega$ be any neighborhood of $\partial \Omega$ not containing $x_{0}$. Then we have

$$
\left\|u_{\varepsilon}\right\| v_{\varepsilon} \rightarrow-2(N-2)(N-4) \sigma_{N} b G\left(\cdot, x_{0}\right) \quad \text { in } \quad C^{3}(\omega) .
$$


Proof. We see

$$
\Delta^{2}\left(\left\|u_{\varepsilon}\right\| v_{\varepsilon}\right)=\left\|u_{\varepsilon}\right\| c_{0} p_{\varepsilon} u_{\varepsilon}^{p_{\varepsilon}-1} v_{\varepsilon}=: f_{\varepsilon}(x)
$$

for $x \in \Omega$ with the boundary condition

$$
\left\|u_{\varepsilon}\right\| v_{\varepsilon}=\Delta\left(\left\|u_{\varepsilon}\right\| v_{\varepsilon}\right)=0 \quad \text { on } \quad \partial \Omega .
$$

By using (2.14), (3.7), (2.9) and the dominated convergence theorem, we obtain

$$
\begin{aligned}
\int_{\Omega} f_{\varepsilon}(x) d x & =c_{0} p_{\varepsilon}\left\|u_{\varepsilon}\right\|^{p_{\varepsilon}+1-\left(\frac{p_{\varepsilon}-1}{4}\right) N} \int_{\Omega_{\varepsilon}} \tilde{u}_{\varepsilon}^{p_{\varepsilon}-1}(y) \tilde{v}_{\varepsilon}(y) d y \\
& \rightarrow c_{0} p \int_{\mathbb{R}^{N}} U^{p-1} v_{0} d y=c_{0} p b \int_{\mathbb{R}^{N}} \frac{1-|y|^{2}}{\left(1+|y|^{2}\right)^{N / 2+3}} d y \\
& =c_{0} p b \sigma_{N}\left(\int_{0}^{\infty} \frac{r^{N-1}}{\left(1+r^{2}\right)^{N / 2+3}} d r-\int_{0}^{\infty} \frac{r^{N+1}}{\left(1+r^{2}\right)^{N / 2+3}} d r\right) \\
& =-2(N-2)(N-4) b \sigma_{N} .
\end{aligned}
$$

Note that the integral involving the $a_{j}$ terms of $v_{0}$ must vanish by the oddness of the integrand. Last integral can be computed by the formula

$$
\int_{0}^{\infty} \frac{r^{\alpha}}{\left(1+r^{2}\right)^{\beta}} d r=\frac{\Gamma((\alpha+1) / 2) \Gamma(\beta-(\alpha+1) / 2)}{2 \Gamma(\beta)} .
$$

Furthermore, for any $x \neq x_{0}$, we have by (2.10) and (3.7),

$$
\begin{aligned}
f_{\varepsilon}(x) & \leq C\left\|u_{\varepsilon}\right\| \frac{\left\|u_{\varepsilon}\right\|^{-\left(p_{\varepsilon}-1\right)}}{\left|x-x_{\varepsilon}\right|^{(N-4)\left(p_{\varepsilon}-1\right)}} \frac{\left\|u_{\varepsilon}\right\|}{\left(\left\|u_{\varepsilon}\right\|^{\left(p_{\varepsilon}-1\right) / 4}\left|x-x_{\varepsilon}\right|\right)^{N-4}} \\
& \leq C \frac{\left\|u_{\varepsilon}\right\|^{3-p_{\varepsilon}-\left(p_{\varepsilon}-1\right)(N-4) / 4}}{\left|x-x_{\varepsilon}\right|^{(N-4) p_{\varepsilon}}} \rightarrow 0
\end{aligned}
$$

since $3-p_{\varepsilon}-\frac{p_{\varepsilon}-1}{4}(N-4)=-8 /(N-4)+\varepsilon(N / 4)<0$ for $\varepsilon>0$ small. From these, we confirm that

$$
f_{\varepsilon} \rightarrow-2(N-2)(N-4) \sigma_{N} b \delta_{x_{0}}
$$

in the sense of distributions. On the other hand, from the equation (3.12) with the Navier boundary condition, $L^{p}$-theory of [5] can be applied to get

$$
\left\|-\Delta\left(\left\|u_{\varepsilon}\right\| v_{\varepsilon}\right)\right\|_{C^{1, \alpha}(\omega)} \leq C(\omega)\left(\left\|f_{\varepsilon}\right\|_{L^{1}(\Omega)}+\left\|f_{\varepsilon}\right\|_{L^{\infty}\left(\omega^{\prime}\right)}\right)
$$

where $\omega \subset \subset \omega^{\prime}$ is a neighborhood of $\partial \Omega$ not containing $x_{0}$. Since we have seen that RHS of the above estimate is bounded by a constant independent of $\varepsilon$, AscoliArzelá theorem implies that the function $-\Delta\left(\left\|u_{\varepsilon}\right\| v_{\varepsilon}\right)$ converges to some function in $C^{1, \alpha}$-topology. Finally, (3.13) implies that this limit function is $-2(N-2)(N-$ 4) $\sigma_{N} b \bar{G}\left(x, x_{0}\right)$. 
Proof of Step 1. Putting $z=x_{0}$ in (2.5) and multiplying $\left\|u_{\varepsilon}\right\|^{2}$, we have

$$
\begin{aligned}
\int_{\partial \Omega}\left(\left(x-x_{0}\right) \cdot \nu_{x}\right) & \left(\frac{\partial\left\|u_{\varepsilon}\right\| u_{\varepsilon}}{\partial \nu_{x}}\right)\left(\frac{\partial\left\|u_{\varepsilon}\right\| \bar{v}_{\varepsilon}}{\partial \nu_{x}}\right) d s_{x} \\
+ & \int_{\partial \Omega}\left(\left(x-x_{0}\right) \cdot \nu_{x}\right)\left(\frac{\partial\left\|u_{\varepsilon}\right\| \bar{u}_{\varepsilon}}{\partial \nu_{x}}\right)\left(\frac{\partial\left\|u_{\varepsilon}\right\| v_{\varepsilon}}{\partial \nu_{x}}\right) d s_{x}=0 .
\end{aligned}
$$

By (2.8) and (3.11), the LHS of (3.14) tends to

$$
-8(N-4)^{2}(N-2)^{2} \sigma_{N}^{2} b \int_{\partial \Omega}\left(\left(x-x_{0}\right) \cdot \nu_{x}\right)\left(\frac{\partial G\left(x, x_{0}\right)}{\partial \nu_{x}}\right)\left(\frac{\partial \bar{G}\left(x, x_{0}\right)}{\partial \nu_{x}}\right) d s_{x}
$$

as $\varepsilon \rightarrow 0$. By $(2.2)$, we obtain

$$
-8(N-4)^{3}(N-2)^{2} \sigma_{N}^{2} b R\left(x_{0}\right)=0 .
$$

Since $R\left(x_{0}\right) \neq 0$, we obtain $b=0$.

Proof of Step 2. In this step, we prove $a_{j}=0, j=1,2, \ldots, N$ in (3.6) by using a lemma, which is not in [9].

Lemma 3.3. Assume $b=0$ and $a=\left(a_{1}, \ldots, a_{N}\right) \neq 0$ in (3.6). Then we have

$$
\left.\left\|u_{\varepsilon}\right\|^{\frac{N-2}{N-4}} v_{\varepsilon} \rightarrow 2(N-2) \sigma_{N} \sum_{j=1}^{N} a_{j}\left(\frac{\partial G}{\partial z_{j}}(x, z)\right)\right|_{z=x_{0}}
$$

in $C_{l o c}^{3}\left(\bar{\Omega} \backslash\left\{x_{0}\right\}\right)$.

Proof. Since $-\Delta \bar{v}_{\varepsilon}=c_{0} p_{\varepsilon} u_{\varepsilon}^{p_{\varepsilon}-1} v_{\varepsilon}$ in $\Omega, \bar{v}_{\varepsilon}=0$ on $\partial \Omega$, the Green representation formula implies that

$$
\bar{v}_{\varepsilon}(x)=c_{0} p_{\varepsilon} \int_{\Omega} \bar{G}(x, z) u_{\varepsilon}^{p_{\varepsilon}-1}(z) v_{\varepsilon}(z) d z
$$

for any $x \in \bar{\Omega} \backslash\left\{x_{0}\right\}$, here $\bar{G}(x, z)=-\Delta_{x} G(x, z)$ is the Green function of $-\Delta$ under the Dirichlet boundary condition. By a change of variables, we see

$$
\begin{aligned}
c_{0} p_{\varepsilon} \int_{\Omega} \bar{G}(x, z) & u_{\varepsilon}^{p_{\varepsilon}-1}(z) v_{\varepsilon}(z) d z \\
& =c_{0} p_{\varepsilon}\left\|u_{\varepsilon}\right\|^{p_{\varepsilon}-\left(\frac{p_{\varepsilon}-1}{4}\right) N} \int_{\Omega_{\varepsilon}} \bar{G}\left(x, \frac{y}{\left\|u_{\varepsilon}\right\|^{\frac{p_{\varepsilon}-1}{4}}}+x_{\varepsilon}\right) \tilde{u}_{\varepsilon}^{p_{\varepsilon}-1} \tilde{v}_{\varepsilon}(y) d y .
\end{aligned}
$$

By (2.12) and (3.3), we obtain

$$
\tilde{u}_{\varepsilon}^{p_{\varepsilon}-1} \tilde{v}_{\varepsilon}(y) \rightarrow \sum_{j=1}^{N} a_{j}\left(\frac{\partial}{\partial y_{j}} \frac{-1}{(N+4)} U^{p}(y)\right)
$$

uniformly on compact subsets of $\mathbb{R}^{N}$. 
Now, let us consider the following linear first order PDE

$$
\sum_{j=1}^{N} a_{j} \frac{\partial w}{\partial y_{j}}=\tilde{u}_{\varepsilon}^{p_{\varepsilon}-1} \tilde{v}_{\varepsilon}(y), \quad y \in \mathbb{R}^{N}
$$

with the initial condition $\left.w\right|_{\Gamma_{a}}=\frac{-1}{(N+4)} U^{p}(y)$, where $\Gamma_{a}=\left\{x \in \mathbb{R}^{N} \mid x \cdot a=0\right\}$. Here, the right hand side is assumed to be 0 outside of $\Omega_{\varepsilon}$. By Lemma 2.7, we have a solution $w_{\varepsilon}$ of this problem with the estimate $w_{\varepsilon}(y)=O\left(|y|^{-(N+3)}\right)$ as $|y| \rightarrow \infty$, since $\tilde{u}_{\varepsilon}^{p_{\varepsilon}-1} \tilde{v}_{\varepsilon}(y)=O\left(|y|^{-(N+4)}\right)$ by (2.14) and (3.7). Also we have

$$
w_{\varepsilon} \rightarrow \frac{-1}{(N+4)} U^{p} \quad \text { uniformly on compact subsets on } \mathbb{R}^{N}
$$

and

$$
\int_{\Omega_{\varepsilon}} w_{\varepsilon}(y) d y \rightarrow \frac{-1}{(N+4)} \int_{\mathbb{R}^{N}} U^{p} d y=\left(\frac{-1}{N+4}\right)\left(\frac{2 \sigma_{N}}{N(N+2)}\right)
$$

by the dominated convergence theorem. Using integration by parts, we have

$$
\begin{aligned}
\bar{v}_{\varepsilon}(x)= & c_{0} p_{\varepsilon}\left\|u_{\varepsilon}\right\|^{p_{\varepsilon}-\left(\frac{p_{\varepsilon}-1}{4}\right) N} \int_{\Omega_{\varepsilon}} \bar{G}\left(x, \frac{y}{\left\|u_{\varepsilon}\right\|^{\frac{p_{\varepsilon}-1}{4}}}+x_{\varepsilon}\right) \sum_{j=1}^{N} a_{j} \frac{\partial w_{\varepsilon}}{\partial y_{j}} d y \\
= & -c_{0} p_{\varepsilon}\left\|u_{\varepsilon}\right\|^{p_{\varepsilon}-\left(\frac{p_{\varepsilon}-1}{4}\right) N} \sum_{j=1}^{N} a_{j} \int_{\Omega_{\varepsilon}} \frac{\partial}{\partial y_{j}}\left\{\bar{G}\left(x, \frac{y}{\left\|u_{\varepsilon}\right\|^{\frac{p_{\varepsilon}-1}{4}}}+x_{\varepsilon}\right)\right\} w_{\varepsilon}(y) d y \\
= & -c_{0} p_{\varepsilon}\left\|u_{\varepsilon}\right\|^{p_{\varepsilon}-\left(\frac{p_{\varepsilon}-1}{4}\right) N-\left(\frac{p_{\varepsilon}-1}{4}\right)} \\
& \times\left.\sum_{j=1}^{N} a_{j} \int_{\Omega_{\varepsilon}}\left(\frac{\partial \bar{G}}{\partial z_{j}}\right)(x, z)\right|_{z=\frac{y}{\left\|u_{\varepsilon}\right\| \frac{p_{\varepsilon}-1}{4}}+x_{\varepsilon}} w_{\varepsilon}(y) d y .
\end{aligned}
$$

Note that $p_{\varepsilon}-\left(\frac{p_{\varepsilon}-1}{4}\right) N-\left(\frac{p_{\varepsilon}-1}{4}\right)=-\left(\frac{N-2}{N-4}\right)+\varepsilon\left(\frac{N-3}{4}\right)$. Thus we obtain

$$
\begin{aligned}
\left\|u_{\varepsilon}\right\|^{\frac{N-2}{N-4}} \bar{v}_{\varepsilon}(x) & \rightarrow-\left.c_{0} p\left(\frac{-1}{N+4}\right)\left(\frac{2 \sigma_{N}}{N(N+2)}\right) \sum_{j=1}^{N} a_{j}\left(\frac{\partial \bar{G}}{\partial z_{j}}(x, z)\right)\right|_{z=x_{0}} \\
& =\left.2(N-2) \sigma_{N} \sum_{j=1}^{N} a_{j}\left(\frac{\partial \bar{G}}{\partial z_{j}}(x, z)\right)\right|_{z=x_{0}}
\end{aligned}
$$

for $x \in \bar{\Omega} \backslash\left\{x_{0}\right\}$.

Standard elliptic estimate assures that this convergence also holds in $C_{\text {loc }}^{1}(\bar{\Omega} \backslash$ $\left.\left\{x_{0}\right\}\right)$. Since $\bar{v}_{\varepsilon}(x)=-\Delta v_{\varepsilon}(x)$, again elliptic estimate proves Lemma 3.3.

Now, assume the contrary that $a=\left(a_{1}, \ldots, a_{N}\right) \neq 0$. We multiply both sides of (2.6) in Lemma 2.4 by $\left\|u_{\varepsilon}\right\|^{(N-2) /(N-4)} \times\left\|u_{\varepsilon}\right\|$ to get

$$
\int_{\partial \Omega}\left(\frac{\partial\left\|u_{\varepsilon}\right\| \bar{u}_{\varepsilon}}{\partial x_{i}}\right)\left(\frac{\partial\left\|u_{\varepsilon}\right\|^{\frac{N-2}{N-4}} v_{\varepsilon}}{\partial \nu_{x}}\right) d s_{x}+\left(\frac{\partial\left\|u_{\varepsilon}\right\| u_{\varepsilon}}{\partial x_{i}}\right)\left(\frac{\partial\left\|u_{\varepsilon}\right\|^{\frac{N-2}{N-4}} \bar{v}_{\varepsilon}}{\partial \nu_{x}}\right) d s_{x}=0 .
$$


As $\varepsilon \rightarrow 0$, we see that the LHS of the above tends to

$$
\begin{aligned}
4(N-4)(N-2)^{2} \sigma_{N}^{2} \sum_{j=1}^{N} a_{j} \times & \int_{\partial \Omega}\left\{\left.\frac{\partial \bar{G}}{\partial x_{i}}\left(x, x_{0}\right) \frac{\partial}{\partial \nu_{x}}\left(\frac{\partial G}{\partial z_{j}}\right)(x, z)\right|_{z=x_{0}}\right. \\
& \left.+\left.\frac{\partial G}{\partial x_{i}}\left(x, x_{0}\right) \frac{\partial}{\partial \nu_{x}}\left(\frac{\partial \bar{G}}{\partial z_{j}}\right)(x, z)\right|_{z=x_{0}}\right\} d s_{x} \\
= & \left.4(N-4)(N-2)^{2} \sigma_{N}^{2} \sum_{j=1}^{N} a_{j} \frac{\partial^{2} R}{\partial z_{i} \partial z_{j}}(z)\right|_{z=x_{0}}
\end{aligned}
$$

here we have used Theorem 2.5 (2.8), Lemma 3.3 and Lemma 2.2 (2.4). Thus

$$
\sum_{j=1}^{N} a_{j} \frac{\partial^{2} R}{\partial z_{i} \partial z_{j}}\left(x_{0}\right)=0 .
$$

Since we assumed that $a=\left(a_{1}, \ldots, a_{N}\right) \neq 0$, this contradicts to our assumption that $x_{0}$ is a nondegenerate critical point of the Robin function. Thus we have proved Step 2 .

Proof of Step 3. By Step 1 and Step 2, we have obtained that the limit function $\lim _{\varepsilon \rightarrow 0} \tilde{v}_{\varepsilon}=v_{0} \equiv 0$. Since $\left\|\tilde{v}_{\varepsilon}\right\|_{L^{\infty}\left(\Omega_{\varepsilon}\right)}=1$, there exists $y_{\varepsilon} \in \Omega_{\varepsilon}$ such that $\tilde{v}_{\varepsilon}\left(y_{\varepsilon}\right)=$ 1 and $\left|y_{\varepsilon}\right| \rightarrow \infty$, because the above convergence $\tilde{v}_{\varepsilon} \rightarrow v_{0} \equiv 0$ is uniform on compact sets of $\mathbb{R}^{N}$. But this is not possible because of Lemma 3.1. This proves Theorem 1.1.

\section{Acknowledgements}

Part of this work was supported by JSPS Grant-in-Aid for Scientific Research, No. 20540216.

\section{References}

[1] T. Bartsch, T. Weth, and M. Willem: A Sobolev inequality with remainder term and critical equations on domains with topology for the polyharmonic operator, Calc. Var. and PDE. 18 (2003) 253-268.

[2] M. Ben Ayed and K. El Mehdi: On a biharmonic equation involving nearly critical exponent, NoDEA 13 (2006) 485-509.

[3] G. Caristi and E. Mitidieri: Harnack inequality and applications to solutions of biharmonic equations, Operator Theory: Advances and Appl. 168 (2006) 1-26.

[4] K. S. Chou and D. Geng: Asymptotics of positive solutions for a biharmonic equation involving critical exponent, Diff. Int. Eq. 13 (2000) 921-940.

[5] D. E. Edmunds, D. Fortunato, and E. Jannelli: Critical exponents, critical dimensions and the biharmonic operator, Arch. Rational Mech. Anal. 112 (1990) 269-289. 
[6] K. El Mehdi: Single blow-up solutions for a slightly subcritical biharmonic equation, Abstr. Appl. Anal. 2006 (2006) Article ID 18387, 20 page.

[7] D. Geng: On blow-up of positive solutions for a biharmonic equation involving nearly critical exponent, Comm. Partial Diff. Eq. 24 (1999) 2333-2370.

[8] D. Geng: Location of the blow up point for positive solutions of a biharmonic equation involving nearly critical exponent, Acta Mathematica Scientia, Ser.B 25B (2005) 283295.

[9] M. Grossi: A nondegeneracy result for some nonlinear elliptic equation, NoDEA 12 (2005) 227-241.

[10] Y. Y. Li: Prescribing scalar curvature on $\mathbb{S}^{n}$ and related problems, Part I, J. Differential Eq. 120 (1995) 319-410.

[11] C. S. Lin: A classification of solutions of a conformally invariant fourth order equation in $\mathbb{R}^{n}$, Comment. Math. Helv. 73 (1998) 206-231.

[12] F. Takahashi: Asymptotic nondegeneracy of least energy solutions to an elliptic problem with the critical Sobolev exponent, Adv. Nonlinear Stud., to appear.

Futoshi Takahashi

Department of Mathematics

Graduate School of Science

Osaka City University

3-3-138 Sugimoto, Sumiyoshi-ku

Osaka, 558-8585

Japan

e-mail: futoshi@sci.osaka-cu.ac.jp

Received: 23 January 2008.

Accepted: 12 May 2008. 